\title{
Electroviscous Effects in Stationary Solid Phase Suspensions
}

\author{
Francisco J. Rubio-Hernández (D)
}

Departamento de Física Aplicada II, Universidad de Málaga, 29071-Málaga, Spain; fjrubio@uma.es

check for updates

Citation: Rubio-Hernández, F.J. Electroviscous Effects in Stationary Solid Phase Suspensions. Fluids 2021, 6, 69. https://doi.org/10.3390/ fluids6020069

Academic Editor: Rajinder Pal

Received: 19 January 2021

Accepted: 2 February 2021

Published: 5 February 2021

Publisher's Note: MDPI stays neutral with regard to jurisdictional claims in published maps and institutional affiliations.

Copyright: (C) 2021 by the author. Licensee MDPI, Basel, Switzerland. This article is an open access article distributed under the terms and conditions of the Creative Commons Attribution (CC BY) license (https:// creativecommons.org/licenses/by/ $4.0 /)$.

\begin{abstract}
Flowing through porous media is a matter of interest in different research fields such as medicine, engineering and science. The spontaneous appearance of ionic distribution at the solid liquid interface gives place to a reduction in the flow rate, which is generally named electroviscous effect. However, this should be differentiated in two more specific effects, the primary effect due to the distortion of ionic clouds, and the secondary effect due to the overlapping of ionic clouds. Theoretical and experimental works have not always been clearly conducted in order to separate both effects. Instead, they have been globally grouped. The purpose of this review is to revise theoretical and experimental bibliography on the electroviscous effect in stationary solid phase suspensions (porous plugs, membranes, microchannels, capillaries). The main conclusions of this brief revision are: (i) when ionic clouds are relatively small, it is possible to accept that only the primary effect is the cause for the apparent increase of the viscosity of the liquid phase when it is forced to flow relative to the stationary solid phase; (ii) although theory predicts a maximum for the variation of the overall electroviscous effect vs the relative size of the ionic cloud, it has been experimentally observed but not properly reasoned that its existence depends on the salt type; and (iii) it is necessary to justify why, if the fluid is non-Newtonian, electrokinetic parameters dominate the characteristics of the flow due to high pressure gradients, but the rheological parameters are more decisive when the flow is generated by low pressure gradients.
\end{abstract}

Keywords: electroviscous effects; suspensions; electrical double layer; electrokinetic phenomena

\section{Introduction}

The study of liquid flow through porous media is of great interest for practical applications in a variety of fields like medicine, engineering and science. A deviation from the normal liquid flow with respect to the stationary solid phase results from the existence of an ionic charge distribution in the vicinity of the solid surface. This ionic charge distribution generates spontaneously when solid and liquid phases contact. Its existence is not an exception and, consequently, it cannot be ignored. Although it has been a matter of interest since 1960's and more specifically during the last decade, some unsolved basic and specific questions need to be answered. These refer to some theoretical predictions and also to the necessity to clearly define the experimental conditions that allow us to differentiate between the primary and the secondary effects that are jointly considered with term electroviscous effect.

The aim of this minireview is to briefly and concisely presents the state of the art on theoretical and experimental results relative to the electroviscous effect observed in suspensions when the liquid phase moves with respect to the stationary solid phase. Parts 2-4 guide the reader towards the main content of the manuscript, which is dealt with in part 5. Some concluding remarks are listed in part 6.

\section{The Viscosity of Hard-Particles Suspensions}

When the size of solid particles dispersed in a base fluid is small enough compared with the dimensions of the vessel that contains the suspension, it can be considered as a homogeneous medium. Assuming the accomplishment of this physical condition, the viscosity of the suspension $(\eta)$ can be defined and calculated as the quotient between the 
shear stress and the shear rate, and then it can be compared with the viscosity of the liquid phase $\left(\eta_{0}\right)$. In fact, when particles are spherical and the suspension is diluted, i.e., when each individual particle does not affect to another or the volume occupied by particles is enough small for the accomplishment of that condition, the viscosity of the suspension can be calculated [1-3]. Einstein considered the perturbation that a single particle (Figure 1b) provokes on the original low-Reynolds-flow-field or Stokes flow existing in the unperturbed liquid phase (Figure 1a).

$$
\eta=\eta_{o}(1+2.5 \phi)
$$

In Equation (1) $\phi$ is the volume fraction occupied by solid particles. Using other approaches, Jeffery [4], Burgers [5], Happel and Brenner [6] obtained the same result. It is necessary to emphasize that Equation (1) is strictly valid only for monodisperse spherical suspensions and low Reynolds number.

Using a cell model, according to which the radius of influence of a particle is higher than its solid radius, Simha [7] calculated the dependence on the solid volume fraction of the viscosity of a monodisperse and concentrated suspension of spherical particles. The asymptotic expression for dilute suspensions $(\phi \rightarrow 0)$, was slightly different from Einstein's result.

$$
\eta=\eta_{o}(1+5.5 \phi)
$$

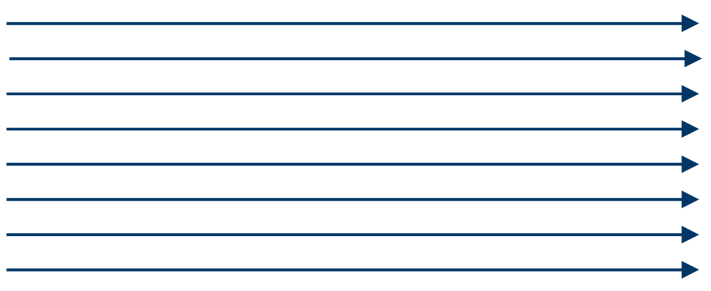

(a)

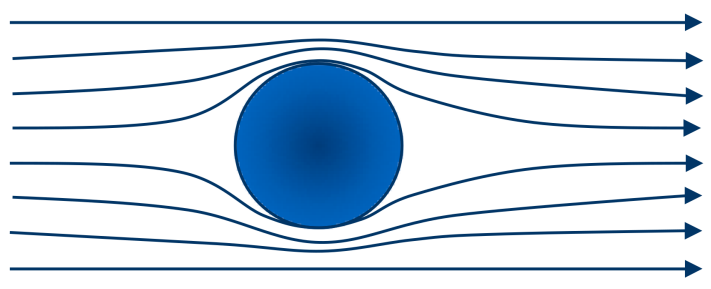

(b)

Figure 1. (a) Laminar flow field in a liquid medium. (b) Distortion in the laminar flow field due to the presence of a single spherical particle.

Equations (1) and (2) can be generalized to include dilute suspensions of particles with shapes other than the spherical one. For that, we will consider [8] the definition of intrinsic viscosity.

$$
[\eta]=\lim _{\phi \rightarrow 0} \frac{\frac{\eta}{\eta_{o}}-1}{\phi}
$$

Therefore, we can write, in general, for dilute suspensions of particles with any shape.

$$
\eta=\eta_{o}(1+[\eta] \phi)
$$

When the particle volume fraction increases to values higher than $10 \%$ but smaller than $20 \%$, which determines the so named moderately concentrated suspensions, the average distance between particles is around the same size as the particle diameter. In this specific case, the flow field around each individual particle is slightly influenced by the presence of neighbor particles, and the rate of energy dissipation when the suspension is forced to flow increases by an amount that is proportional to the square of $\phi$. This new situation gives place to a quadratic dependence of the viscosity with the solid volume fraction.

$$
\eta=\eta_{o}\left(1+[\eta] \phi+C \phi^{2}\right)
$$

The first calculation of $C$ was published only in 1972 [9]. This long wait was due to the complexity of the flow field that is generated between particles, even in the simplest case of only two spheres with the same radius [10]. Batchelor and Green [9] obtained C $=7.6$ for a 
non-Brownian moderately concentrated monodisperse suspension of hard spheres. When the Brownian motion is taken into account, a different result, $C=6.0$, is obtained [11].

If the solid volume fraction increases beyond $20 \%$, many-body hydrodynamic interactions must be considered. Therefore, a huge unsolved theoretical challenge is still in front of theoreticians. A manner to approximate to the solution, particle simulation methods such as Stokesian [12] lattice Boltzmann [13], or dissipative particle dynamics [14] have been used. Alternatively, semi-empirical (therefore also approximate) equations have been proposed to describe the dependence of the viscosity of suspensions in the whole range of solid volume fraction, i.e., including concentrated suspensions. However, it is worthy to note that fine comparison to experimental results is really problematic because it is very difficult to achieve a monodisperse concentrated suspension of hard spheres for accurate testing. Following with the description of this approach, the most popular semiempirical equations for the dependence of the viscosity of a concentrated suspension with the solid volume fraction are those proposed by Krieger and Dougherty [15],

$$
\eta=\eta_{o}\left(1-\frac{\phi}{\phi_{m}}\right)^{-[\eta] \phi_{m}}
$$

Quemada [16],

$$
\eta=\eta_{o}\left(1-\frac{\phi}{\phi_{m}}\right)^{-2}
$$

and Mooney [17].

$$
\eta=\eta_{o} \exp \left(\frac{[\eta] \phi}{1-\frac{\phi}{\phi_{m}}}\right)
$$

In these equations $\phi_{m}$ is the maximum packing fraction, which expresses the ability of the particles to achieve the most compact distribution. It should be noted that in these proposals no dependence of the suspension viscosity with shear (rate or stress) is assumed. In other words, these expressions were formulated assuming Newtonian behavior of the suspension in the whole solid volume fraction interval despite that, as it is well known, concentrated suspensions show a variety of non-Newtonian behaviors. This is, consequently, a failure of these semiempirical approaches that clearly needs to be solved. Some attempts have been made to complete these equations including empiric shear rate dependencies. For example, Gómez-Merino et al. [18] obtained the shear rate dependence of $[\eta]$ and $\phi_{m}$ in Equations (6) and (8) using data of the relative viscosity of anatase water nanofluids in the low and high shear rate limits.

\section{The Electrical Double Layer}

It is an extensively observed experimental fact that, in most of the cases, when solid particles are put in contact with a polar liquid phase, an excess of electrical charge appears on the solid surface. This surface charge provokes an additional distribution of ions in the volume that surrounds the particle. Charges of the same sign (co-ions) are repelled from the particle surface, while charges of the opposite sign (counter-ions) are attracted to it. Random thermal agitation tends to mix the ions in the liquid phase giving place to the formation of a diffuse electrical layer that, with the surface charge originally formed on the solid particle surface, a charge configuration named electrical double layer (EDL) develops [19].

Obviously, the distribution of ions in the EDL is directly related to the electric potential value on the charged solid surface $\left(\psi_{0}\right)$, which is measured with respect to the zeroreference value in the liquid bulk, where the electric neutrality is logically achieved. The first model relating charge distribution and electric potential in the EDL was developed by Helmholtz in 1879 [20]. In Helmholtz's model the EDL is assumed small enough to be represented by a parallel plate capacitor with a gap $\kappa^{-1}$ (named Debye length) between 
plates (Figure 2a). In this case, the potential decreases linearly with the distance $x$ to the particle surface.

$$
\psi=\psi_{o}(1-\kappa x)
$$

Later, and independently, Gouy [21] and Chapman [22] developed a second model that also considers the thermal agitation of both types of ions in the liquid phase (Figure $2 b$ ). Consequently, the "plate of the capacitor" representing the charge in the liquid phase is really a diffuse layer (DL) of charge that results from the equilibrium state achieved by ions when both, the electric field and the thermal agitation, are simultaneously considered. The DL extends a distance $d$ towards the bulk of the liquid phase. According to the GouyChapman model, the potential decreases with the distance to the particle surface following a much more complicated function,

$$
\psi=\frac{2 k T}{z e} \ln \left[\frac{1+A \exp (-\kappa x)}{1-A \exp (-\kappa x)}\right]
$$

where,

$$
A=\frac{\exp \left(z e \psi_{0} / 2 k T\right)-1}{\exp \left(z e \psi_{0} / 2 k T\right)+1}
$$

and $e$ is the elementary charge, $k$ the Boltzmann constant, and $T$ the absolute temperature.

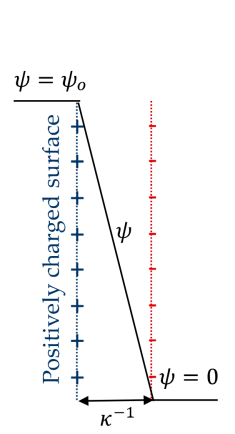

(a)

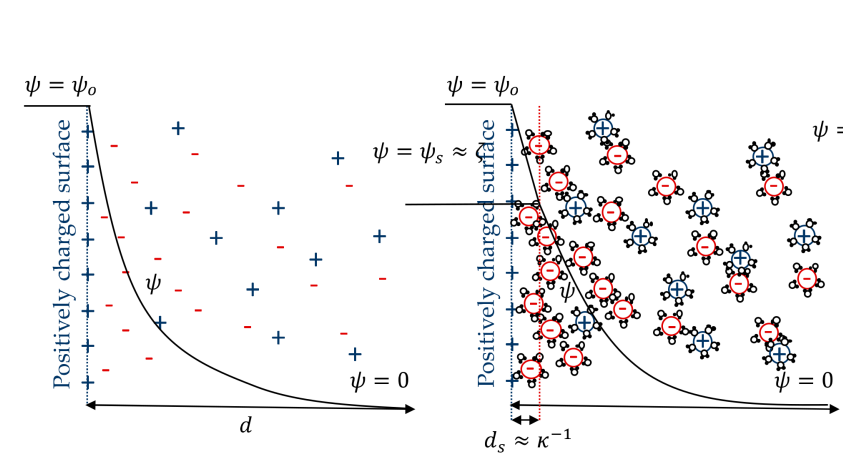

(c)

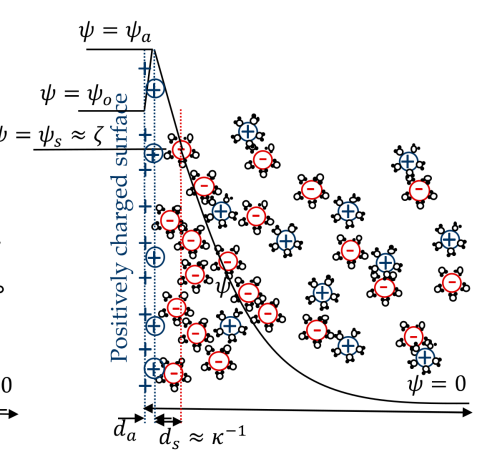

(d)

Figure 2. Models of the electrical double layer: (a) Helmholtz, (b) Gouy and Chapman, (c) Stern and Grahame, and (d) Stern and Grahame with specific adsorption of co-ions.

Stern [23] and Grahame [24] modified the Gouy-Chapman model by considering the finite size of ions and the adsorption or solvation forces of chemical origin that gives place to an ion hydrodynamic radius larger than the radius of "dry" ions. Therefore, counter-ions can maintain a finite distance $d_{s}$ from the particle surface (Figure 2c). There the potential takes the value $\psi_{s}$, and the Gouy-Chapman model for the diffuse layer can be used substituting $\psi_{o}$ by $\psi_{s}$. It is a good approximation to assume that the mobile part of the EDL starts at the distance $d_{s}$, in which the slipping plane is localized. Therefore, it coincides with the Debye length. This electric potential, that can be correlated with the wettability in porous media [25], is measurable with electrokinetics methods and takes the specific name of zeta potential $(\zeta)$. The Stern-Grahame model can be refined by including the formation of a surface layer of thickness $d_{a}$ that consists of specific adsorbed co-ions (Figure $2 \mathrm{~d}$ ). At a distance $d_{a}$ of the particle surface the potential takes a value $\psi_{a}>\psi_{o}$. The potential decreases until a value $\zeta$ at a distance $\kappa^{-1}$, which defines the slipping plane from where the mobile part of the EDL starts.

If the relative movement of the parts separated by the slipping plane is forced, a series of electrokinetic phenomena appear. These can be used to obtain the $\zeta$-potential value. Therefore, a brief description of the classical electrokinetic phenomena will be given now. 


\section{Electrokinetic Phenomena}

The relative movement at the slipping plane, where the electric potential in the EDL takes the value $\psi=\zeta$, can be provoked by an external action (electric field, pressure gradient, gravitational field). This mechanism defines the so-called electrokinetic phenomena, being electro-osmosis, electrophoresis, streaming potential, and sedimentation potential, the classical ones. Therefore, the use of experimental electrokinetic methods can lead to calculate the value of $\zeta$-potential [26].

If an electric field $(E)$ provokes a movement of the liquid phase parallel to the stationary solid surface plane, the electrokinetic phenomenon is called Electro-osmosis. The movement of the liquid phase is caused by the drag of counter-ions that are more abundant near the solid surface, which is opposite charged. The maximum velocity of the liquid phase with respect to the stationary solid phase $\left(v_{e o}\right)$ can be measured and related to the $\zeta$-potential using Smoluchowski's original theory [27].

$$
\frac{v_{e o}}{E}=\mu_{e o}=-\frac{\varepsilon}{\eta} \zeta
$$

In Equation (12) $\varepsilon$ is the dielectric constant of the liquid phase, $\eta$ its viscosity and $\mu_{e o}$ the electro-osmotic mobility.

If an electric field provokes the movement of the solid particles with a velocity $v_{e}$ with respect to the stationary liquid phase, the electrokinetic phenomenon is called Electrophoresis. Again, the simplest calculation of the relationship between the velocity of the particles and $\zeta$-potential was derived by Smoluchowski assuming that the electric field was parallel to the particle surface [26].

$$
\frac{v_{e}}{E}=\mu_{e}=\frac{\varepsilon}{\eta} \zeta
$$

In Equation (13) $\mu_{e}$ is the electrophoretic mobility. It is worthy to note that Smoluchowski's analysis assumes the EDL is very small compared to the solid particle size. In other words, if $a$ is the particle radius, Smoluchowski's derivations are valid with the accomplishment of the condition $\kappa a \gg 1$. Hückel [28] obtained a relationship between the electrophoretic mobility and $\zeta$ when the opposite condition is accomplished $(\kappa a \ll 1)$, i.e., when the EDL is thick compared to the particle size.

$$
\mu_{e}=\frac{2}{3} \frac{\varepsilon}{\eta} \zeta
$$

For his derivation, Hückel neglected the distortion of the electric field provoked by the presence of particles. Therefore, when Henry [29] considered in his calculations the alteration of the electric field in the vicinity of solid particles, he obtained a general expression that asymptotically tends to Smoluchowski and Hückel equations in the limits $\kappa a \gg 1$ and $\kappa a \ll 1$, respectively.

$$
\mu_{e}=\frac{2}{3} \frac{\varepsilon}{\eta} \zeta f(\kappa a)
$$

In Equation (15) $f(\kappa a)$ is a correction factor called the Henry's function. Logically, $f(\kappa a)=\frac{3}{2}$ for $\kappa a \gg 1$ and $f(\kappa a)=1$ for $\kappa a \ll 1$.

If the movement of the liquid phase with respect to the stationary solid particles that form a porous plug is provoked by a pressure gradient $(\Delta P)$, the electrokinetic phenomenon is called Streaming potential. The movement of the liquid phase provokes a distortion of the EDL and, consequently, the development of a potential difference between the porous plug extremes. This electric potential difference is also named streaming potential $(\Delta \phi)$. The first relationship between streaming potential and $\zeta$-potential was obtained by Helmholtz [20], and later by Smoluchowski [30], assuming laminar flow, absence of 
surface conductance, and narrow EDL compared to the average radius of the porous plug $(\kappa a \gg 1)$ [26]. $a$ refers now to the radius of the porous plug.

$$
\zeta=\left(\frac{\Delta \phi}{\Delta P}\right) \frac{\eta}{\varepsilon} \lambda
$$

In Equation (16) $\lambda$ is the specific electrical conductivity of the suspension. Equation (16) was extended to any $\kappa a$ value and small $\zeta$-potentials by Rice and Whitehead [31], and any $\kappa a$ value and $\zeta$-potentials by Levine et al. [32]. These authors added a corrective factor to Equation (16),

$$
\zeta=\left(\frac{\Delta \phi}{\Delta P}\right) \frac{\eta}{\varepsilon} \lambda \frac{1}{F(\kappa a)}
$$

where,

$$
F(\kappa a)=\frac{1-\frac{2 I_{1}(\kappa a)}{\kappa a I_{0}(\kappa a)}}{1-\frac{1}{\lambda \eta}\left(\frac{\varepsilon \kappa k T}{e}\right)^{2}\left[1-\frac{2 I_{1}(\kappa a)}{\kappa a I_{0}(\kappa a)}-\left(\frac{I_{1}(\kappa a)}{I_{0}(\kappa a)}\right)^{2}\right]}
$$

was the value obtained by Rice and Whitehead for the corrective factor. In Equation (18), $I_{0}$ and $I_{1}$ are the Bessel functions of 0 and 1 order, respectively. On the other hand, Levine et al. [32] proposed a different expression for the corrective factor when extended the applicability of Equation (17) to any $\zeta$-potential value, which could justify the best agreement of this approach with some experimental results [33].

$$
F(\kappa a)=\frac{1}{2} \frac{(\kappa a)^{2}\left(1-\frac{2}{(\kappa a)^{2} \psi_{s}} \int_{0}^{\kappa a} r \psi(r) d r\right)}{\int_{0}^{\kappa a} r \cosh \psi(r) d r+\frac{1}{\lambda \eta}\left(\frac{\varepsilon \kappa k T}{e}\right)^{2} \int_{0}^{\kappa a} r\left(\frac{d \psi(r)}{d r}\right)^{2} d r}
$$

In Equation (19) $\psi(r)$ is the electric potential at a distance $r$ from the particle surface and $\psi_{s}$ the electric potential value at the particle surface $(r=0)$.

If the solid particles sediment due to the action of gravity, a displacement of counterions from front to back occurs giving place to an electric current equal, in absolute terms, to the current generated down by the charge that exists onto the particle surface. The equality of both currents in the steady state leads to the appearance of an electric field $(E)$ named Sedimentation potential, which is related to the $\zeta$-potential.

$$
\zeta=\frac{3 \eta \lambda_{0}}{8 \pi \varepsilon_{0} \varepsilon a^{3}\left(\rho_{s}-\rho_{l}\right) g n(1+\kappa a)} E
$$

In Equation (20) $\lambda_{0}$ is the bulk conductivity, $\varepsilon_{0}$ the permittivity of the vacuum, $\rho_{s}$ the density of the solid phase, $\rho_{l}$ the density of the liquid medium, $g$ the gravitational field, and $n$ the number of particles per unit volume [26].

All these models that relate $\zeta$-potential with some macroscopic magnitude that is experimentally accessible, have been progressively improved considering the influence of the type of ions, the shape of particles, the mobility of ions near the particle surface, etc. However, it is not necessary for our purpose to revise that deeper description of the electrokinetic phenomena. It is enough in this context to realize that a microscopic magnitude that characterizes the EDL, i.e., the $\zeta$-potential, can be indirectly accessible for the researcher thanks to the use of macroscopic experimental techniques that derive from the existence of electrokinetic phenomena.

\section{Electroviscous Effects}

It is expected that the existence of an EDL that surrounds solid particles has some influence in the flow of suspensions. This must happen when the liquid phase moves with respect to the stationary solid phase or when the solid phase moves with respect to the stationary liquid media. Here we are concerned in the results obtained on the first 
experimental and theoretical design, i.e., when the liquid phase moves with respect to the stationary solid phase. Revisions of the primary electroviscous effect in suspensions when the solid phase moves with respect to the stationary liquid phase can found in $[34,35]$.

If an electrolyte (ionic solution) is forced to flow through a porous plug due to the application of a pressure gradient, the streaming potential which is generated due to the separation of ions of different signs, provokes a backflow of liquid by the electro-osmotic effect (drag due to the movement of co-ions). Therefore, as the flow rate diminishes, the viscosity of the electrolyte is apparently higher as compared to the viscosity of the system in absence of EDL. The deformation of each EDL and not the overlapping of different EDLs will be the most relevant mechanism in the process only if the radius of the porous is large enough compared to the extent of the EDL (Figure 3). It is worthy to note that only in this case we could talk properly about the primary electroviscous effect in these sorts of concentrated suspensions. Therefore, considering surface charge-dependent slip, the Navier-Stokes equation was solved for the flow in a microtube of radius $a$ much larger than the Debye length $(1 / \kappa)$, i.e., for $\kappa a \gg 1$. Consequently, the calculations about the effect of the EDL on the flow rate were in fact referred to as the primary electroviscous effect; despite it not specifically being indicated by the authors [36]. These authors found that the (primary) electroviscous effect increases with the increase of $\zeta$-potential. It is worthy to note that neglecting secondary electroviscous effect due to the overlapping of EDL from different particles when it exists, gives place to mistakes as high as $30 \%$ in the estimation of streaming conductance in microchannels [37]. Consequently, differences between experimental data and theoretical results are higher when the ionic concentration in the liquid phase is smaller because, in this case, the EDL thickness is larger and the consequences of the overlapping of different EDLs are more dramatic. On the other hand, when the ionic strength is relatively high $\left(10^{-2} \mathrm{M} \mathrm{KCl}\right)$ or the $p H$ value is near the isoelectric point, the EDL is small enough $(\kappa a \gg 1)$ to consider the influence of only the primary electroviscous effect. This is an important condition that should be considered for proper interpretation of the results.
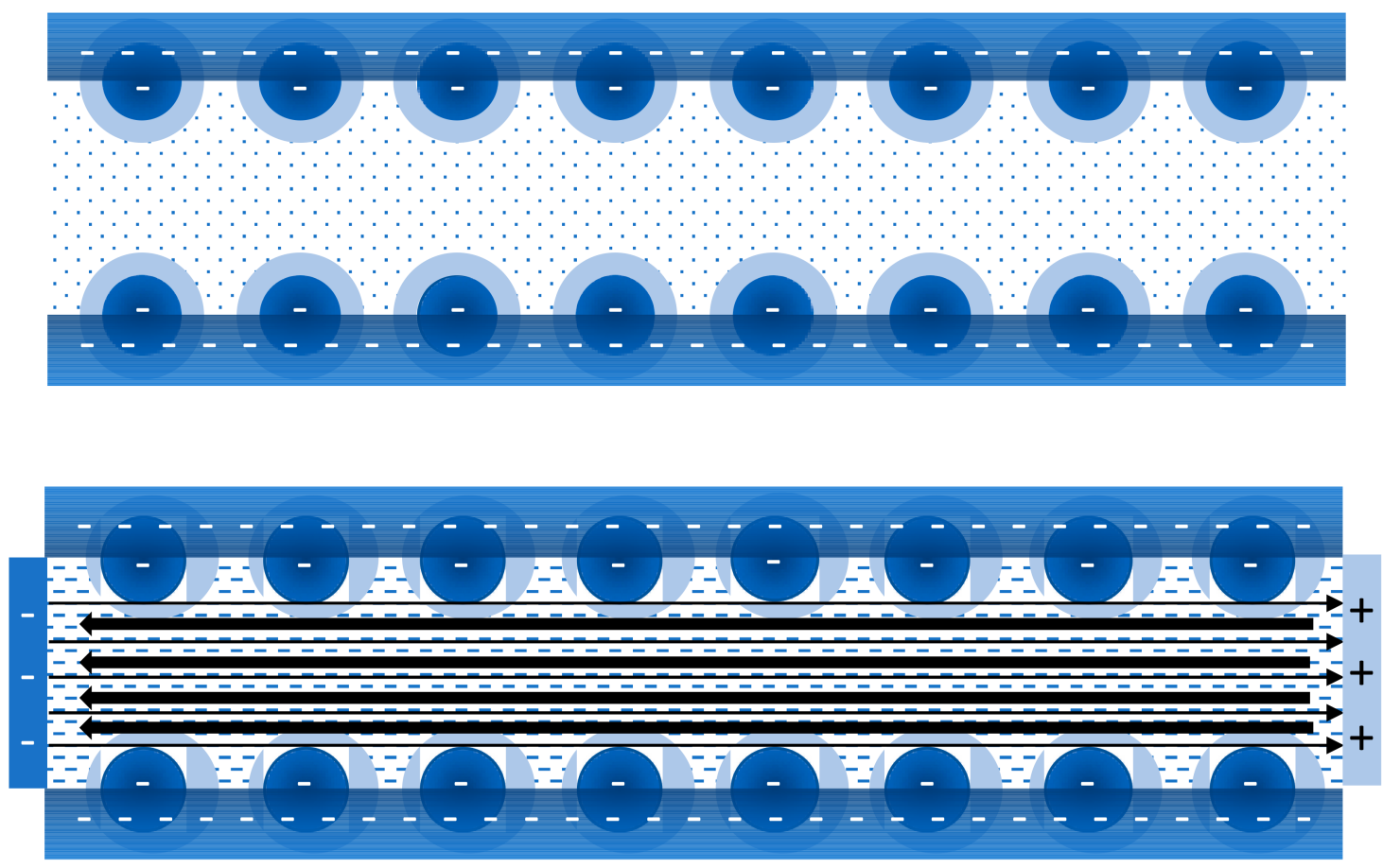

Figure 3. Scheme for the description of the primary electroviscous effect in a porous plug. It is assumed that the sign of the charge developed onto the particle surface is negative. Therefore, the EDL is mainly positive. At rest (figure on the top) the EDL maintains its symmetry. However, when a pressure difference generates a flow along the capillaries of the porous plug from left to right (figure on the bottom), the distortion of the EDL gives place to an excess of positive charge at the right part of the porous plug. Consequently, an electric field pointing from right to left is induced, generating an electro-osmotic effect on the positive ions (counter-ions in this case) that reduces the velocity of the flow, i.e., the viscosity is apparently enhanced. 
Some authors [38] have also reported the increase of the resistance to flow of electrolytes in rectangular microchannels, which is an indication of the existence of electroviscous effects in these sorts of liquid flows. Apparently, their measurements refer to the whole effect that provokes the existence of EDL in solid-liquid interfaces, without discriminating against the separate effects on the liquid flow due to only the distortion (primary electroviscous effect) or considering specifically the overlapping (secondary electroviscous effect) of EDL. However, an analysis of the experimental conditions in which their results were obtained, lead us to conclude that the study was referring to the primary effect. Effectively, the results by Ren et al. [38] correspond to electrokinetic channels with electrokinetic radius $(\mathrm{Ka})$ that varied between 200 to 700 for the $10^{-4} \mathrm{M}(\mathrm{KCl}$ or $\mathrm{LiCl})$ solutions, and between 500 to 1700 for the $10^{-4} \mathrm{M} \mathrm{AlCl}_{3}$ solution. It is clear that, even considering the lowest value $(\kappa a=200)$ the thickness of the EDL $(1 / \kappa)$ is 200 times thinner than the half of the microchannel despite the use of low electrolyte concentration. In other words, only the distortion of EDL must play a significant role in the reduction of the liquid flow or the increase of its apparent viscosity. Results by Ren et al. [38] agree qualitatively with models that predict a decrease of the electroviscous effect with the increase of the ionic strength in the liquid media [39] except for the case of $10^{-4} \mathrm{M} \mathrm{LiCl}$ solutions. With this electrolyte, a maximum in the dependence of the relative viscosity with the electrokinetic radius was found at $\kappa a=400$. The authors did not justify the existence of a maximum in the electroviscous effect that, on the other hand, is predicted (although no explained) by the majority of the theoretical approaches [31,32,40-42]. Therefore, on one hand, it is worthy to establish clearly whether the reduction of the liquid rate flow is due to only the distortion (primary electroviscous effect) of EDL or if the overlapping of different EDLs (secondary electroviscous effect) play a role, and, on the other hand, it is necessary to answer two questions: does the maximum in the electroviscosity vs ionic strength plot depend on the salt type for the same microchannel? If so, why? In addition, to generate more confusion, results of a recent model for the drag flow in microchannels with non-overlapping EDL [43] show that a maximum in the primary electroviscous effect appears only when the flow produces with charge-dependent slip condition. When the flow is accomplished with the non-slip condition, the maximum disappears. So, we think that this result should be considered as a clue to justify previous experimental and theoretical results. Moreover, this idea is supported by a recent study [44] which found that the slip increases the influence of ionic concentration on the dimensionless flow rate.

When the flow of the liquid phase through a porous plug is analyzed from a phenomenological point of view, the non-Equilibrium Thermodynamics establishes linear relationships between generalized forces and fluxes [45] and can supply an efficient way for the study of the electroviscous effect. For porous plugs it is appropriate to consider that the generalized forces are the hydrostatic pressure difference $(\Delta P)$ and the electrical potential difference $(\Delta \phi)$, and the generalized fluxes are the total volumetric flux $(Q)$ and the electric current flux $(J)$. Then, the linear relationships are:

$$
\begin{gathered}
Q=L_{11} \Delta P+L_{12} \Delta \phi \\
J=L_{21} \Delta P+L_{22} \Delta \phi
\end{gathered}
$$

In Equations (21) and (22) $L_{i j}$ are the phenomenological coefficients. From Equation (21) it is clear that $L_{11}$ characterizes the hydrodynamic flow,

$$
L_{11}=\left(\frac{Q}{\Delta P}\right)_{\Delta \phi=0}
$$

i.e., this coefficient can be experimentally obtained from the slope of the linear plot of the total volumetric flux vs. the increasing hydrostatic pressure difference. The condition of lin- 
earity is accomplished when laminar flow is maintained and, according to the relationship obtained by Overbeek [46], a classical result for uncharged Poiseuille flow is obtained.

$$
L_{11}=\frac{n \pi a^{4}}{8 \eta \ell}
$$

If the geometry of the porous plug is constant (i.e, when the number $n$ of capillaries, the average radius $a$, and the length $\ell$ are constants), the variation of $L_{11}$ must be due only to changes in the viscosity of the liquid phase $(\eta)$. As an example of the use of this procedure elsewhere [47], it was obtained that $L_{11}$ increased with the ionic strength in methanol-water mixtures $(50 \% v / v)$ despite the fact that the viscosity of the liquid phase was maintained constant. So, the existence of electroviscous effects was inferred in order to justify this result [48]. To arrive at this conclusion, Levine et al. 's considered the existence of an EDL [32]. Noting that $\left(\frac{\Delta \phi}{\Delta P}\right)_{J=0}=-\left(\frac{Q}{J}\right)_{\Delta P=0^{\prime}}$ as it is deduced from Equations (21) and (22), Levine et al. obtained the apparent viscosity $\left(\eta_{a}\right)$ of the liquid contained into the porous plug.

$$
\eta_{a}=\frac{\eta}{1-\frac{3 C Z^{2}}{2(1+H)(\kappa a)^{2}}}
$$

In Equation (25) $Z=\frac{e \zeta}{k T}$ is the dimensionless $\zeta$-potential, $C=\frac{4 \varepsilon k T N}{\lambda \eta}$ with $N$ the ion concentration and $\lambda$ the electrical conductivity of the liquid phase, and $H=\frac{4(1+C) \sinh ^{2}\left(\frac{Z}{4}\right)}{\kappa a}$. When the electrokinetic radius is high ( $\kappa a \gg 1)$, Equation (25) predicts that $\eta_{a} \approx \eta$, which was experimentally confirmed [48].

Electroviscous effects have also been observed in the flow of electrolytes through fibers [49], ceramic [50], polysulphone [51], and polycarbonate [52] membranes. Experimental results agree with theoretical predictions [31,32,40,41]. However, again the physical justification for the existence of the maximum in the electroviscous effect vs. the electrokinetic radius has not been adequately discussed. While it is clear that the electroviscous effect must decrease when the electrokinetic radius increases due to narrowing of the EDL, it is necessary to justify the decrease of the effect when the electrokinetic radius decreases to very small values, i.e., despite the fact that the EDL largely extends towards the bulk of the liquid phase. On the other hand, other authors have found only monotonous decrease of the electroviscous effect in $\gamma$-alumina membranes by the increasing ionic strength of the liquid media [53]. Therefore, two new questions maintain unanswered: does the maximum in the electroviscosity vs. ionic strength plot depend on membrane composition? If so, why?

The last group of papers on electroviscous effects in stationary solid phase suspensions that will be here referenced, discusses the flow of non-Newtonian liquid phases relative to the stationary solid phase. If the viscosity of the fluid depends on the velocity gradient (shear rate) according to a power law,

$$
\eta=K \dot{\gamma}^{n-1}
$$

where $K$ and $n$ are model parameters, and $\dot{\gamma}$ is the shear rate, the influence of the electroviscous effect on the flow respect to the stationary solid phase has been calculated considering two non-Newtonian viscous behaviors, i.e., shear-thinning $(n<1)$ and shear-thickening $(n>1)$ [54-57]. It was found that the electroviscous effect is dominant when the fluid is shear thinning because the viscosity near the wall is smaller than that corresponding to the Newtonian behavior $(n=1)$. This result has also been confirmed using the Carreau model [58] for the dependence of the viscosity of a shear-thinning fluid with the shear rate.

$$
\eta=\eta_{\infty}+\left(\eta_{o}-\eta_{\infty}\right)\left[1+(m \dot{\gamma})^{2}\right]^{\frac{n-1}{2}}
$$


In Equation (27), $\eta_{0}$ and $\eta_{\infty}$ are limiting viscosity values at low and high shear rates, and $m$ and $n$ are model parameters. On the other hand, due to the increase of the viscosity near the wall when the fluid is shear-thickening as compared to the Newtonian case, the influence of the electroviscous effect is negligible. We suggest an explanation for this behavior. Considering Figure 4, the velocity gradient at the wall developed by a Newtonian fluid can be taken as reference. Therefore, it is clear that the velocity gradient at the wall developed by a power-law shear-thinning is higher, while the velocity gradient at the wall developed by a power-law shear-thickening is lower.

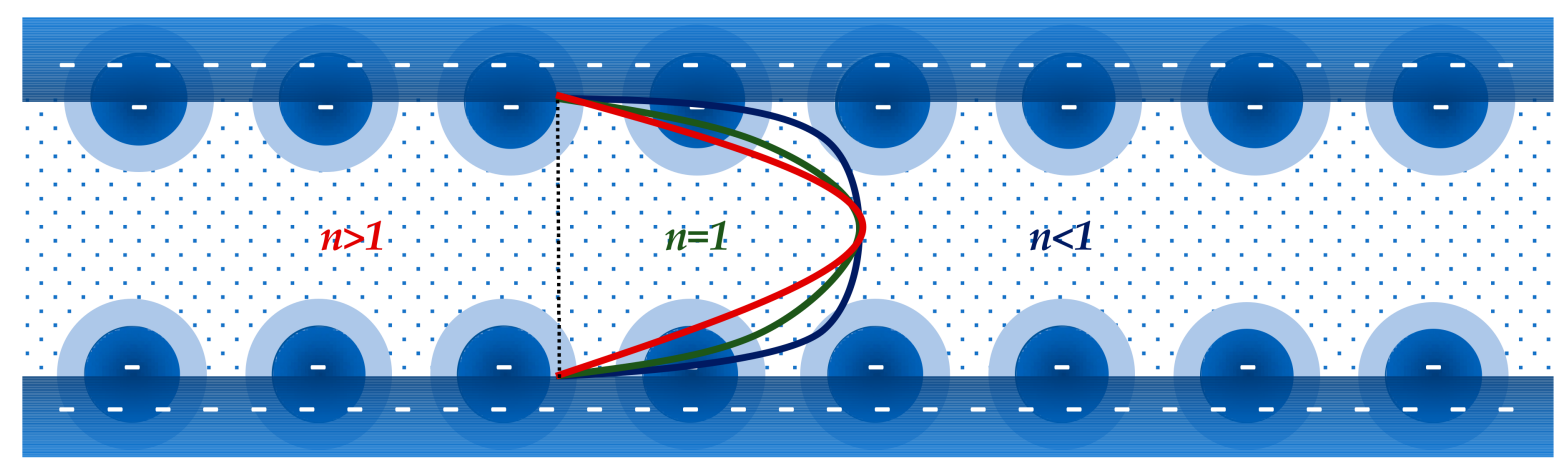

Figure 4. Velocity profiles of shear-thinning $(n<1)$, Newtonian $(n=1)$, and shear-thickening $(n>1)$ fluids.

Also, the influence of the flow index value $(n)$ on the heat transfer in microchannels has been theoretically discussed [59]. Finally, very recently [60], the electroviscous flow of Bingham-Papanastasiou fluids ( $\eta_{0}$ is the plastic viscosity, $\sigma_{y}$ is the yield stress and $m$ is the stress growth index),

$$
\eta=\eta_{0}+\frac{\sigma_{y}}{\dot{\gamma}}[1-\exp (-m \dot{\gamma})]
$$

in circular microtubes has been theoretically studied by solving simultaneously PoissonBoltzmann and the movement (equivalent to Navier-Stokes for Newtonian fluids) equations. The relative influence of electrokinetic ( $\zeta$-potential and Debye length) and rheological $\left(\sigma_{y}\right.$ and $\left.m\right)$ parameters on the non-linear response of the flow has been studied. These authors concluded that electrokinetic parameters dominate the characteristics of the flow when it is provoked by higher pressure gradients, while the rheological parameters are decisive for flows due to low pressure gradients.

\section{Discussion and Further Work}

Theoretical and semiempirical equations for the dependence of the viscosity of concentrated suspensions of hard particles have been formulated assuming Newtonian behavior of the suspension. However, it is well-known that concentrated suspensions usually show non-Newtonian behavior. This failure of the theory needs to be solved.

When measuring electroviscous effects in microchannels, membranes or porous plugs, results on primary and secondary effects are overlapped. Only when the electrokinetic radius $(\kappa a)$ is high enough to assume the EDL is really small, it is possible to accept that only the distortion of the EDL and then, the primary effect, is the cause for the apparent increase of the viscosity of the liquid phase when is forced to flow with respect to the stationary solid phase. Therefore, experimental conditions for the study of each electroviscous effect should be clearly established.

A maximum in the electroviscous effect vs ionic strength has been experimentally observed. This trend is dependent on the salt type for the same microchannel. This surprising observation needs to be justified. It should be taken into account that, related to this observation, a recent model for the drag flow in microchannels with non-overlapping EDL [43] shows that the maximum in the primary electroviscous effect appears when the flow is generated with charge-dependent slip condition but, when the flow is accomplished with the non-slip condition, the maximum disappears. 
Non-Equilibrium Thermodynamics approach is an efficient way for the study of the electroviscous effect that should be extensively explored.

Although the electroviscous effect should decrease when the electrokinetic radius increases, due to the narrowing of the EDL, the decrease of the effect when the electrokinetic radius decreases to very small values has been experimentally observed in membranes, despite the fact that the EDL largely extend towards the bulk of the liquid phase in this case. On the other hand, other authors have found only a monotonous decrease of the electroviscous effect with increasing ionic strength of the liquid media. Therefore, does the maximum in the electroviscosity vs. ionic strength plot depend on membrane composition? If so, why?

It has been observed that the electroviscous effect is dominant when the fluid is shear thinning but negligible when the fluid is shear-thickening as compared to the Newtonian case. These results can be justified noting that the velocity gradient at the wall developed by shear-thinning fluids is higher, and the development by shear-thickening fluids is lower than a Newtonian fluid.

Finally, related to the flow of non-Newtonian fluids respect to stationary solid phases, it has been concluded, that electrokinetic parameters dominate the characteristics of the flow when it is provoked by higher pressure gradients, but the rheological parameters are decisive for flows due to low pressure gradients. Some physical justification for this result is necessary.

Funding: This research received no external funding.

Institutional Review Board Statement: Not applicable.

Informed Consent Statement: Not applicable.

Conflicts of Interest: The author declares no conflict of interest.

\section{References}

1. Einstein, A. Eine neue Bestimmung der Moleküldimensionen. Ann. Phys. 1906, 19, 289-306. [CrossRef]

2. Einstein, A. Berichtigung zu meiner Arbeit: "Eine neue Bestimmung der Moleküldimensionen. Ann. Phys. 1911, 34, 591-592. [CrossRef]

3. Einstein, A. Investigations on the Theory of the BROWNIAN Movement; Dover Publications, Inc.: New York, NY, USA, 1956; pp. 36-54.

4. Jeffery, G.B. The motion of ellipsoidal particles immersed in a viscous fluid. Proc. R. Soc. Lond. A 1922, 102, 161-179.

5. Burgers, J.M. Second Report on Viscosity and Plasticity; North Holland Publishing, Co.: Amsterdam, The Netherlands, $1938 ;$ p. 113.

6. Happel, J.; Brenner, H. The viscosity of particulate systems. In Low Reynolds Number Hydrodynamics, 2nd ed.; Moreau, R.J., Ed.; Martinus Nijhoff Publishers: The Hague, The Netherlands, 1973; pp. 431-473.

7. Simha, R. A treatment of the viscosity of concentrated suspensions. J. Appl. Phys. 1952, 23, 1020-1023. [CrossRef]

8. Dealy, J.M.; Morris, J.; Morrison, F.; Vlassopoulos, D. Official symbols and nomenclature of The Society of Rheology. J. Rheol. 2013, 57, 1047-1055.

9. Batchelor, G.K.; Green, J.T. The determination of the bulk stress in a suspension of spherical particles to order $\mathrm{c}^{2}$. J. Fluid Mech. 1972, 56, 401-427. [CrossRef]

10. Mewis, J.; Wagner, N.J. Colloidal Suspension Rheology; Cambridge University Press.: New York, NY, USA, $2012 ;$ pp. 46-51.

11. Bergenholtz, J.; Brady, J.F.; Vicic, M. The non-Newtonian rheology of dilute colloidal suspensions. J. Fluid Mech. 2002, 456, 239-275. [CrossRef]

12. Brady, J.F.; Bossis, G. Stokesian dynamics. Annu. Rev. Fluid Mech. 1988, 20, 111-157. [CrossRef]

13. Chen, S.; Doolen, G.D. Lattice Boltzmann method for fluid flows. Ann. Rev. Fluid Mech. 1998, 30, 329-364. [CrossRef]

14. Martys, N.S. Study of a dissipative particle dynamics based approach for modeling suspensions. J. Rheol. 2005, 49, 401-424. [CrossRef]

15. Krieger, I.M.; Dougherty, T.J. A mechanism of non-Newtonian flow in suspensions of rigid spheres. Trans. Soc. Rheol. 1959, 3, 137-152. [CrossRef]

16. Quemada, D. Rheology of concentrated disperse systems and minimum energy-dissipation principle: 1 . Viscosity-concentration relationship. Rheol. Acta 1977, 16, 82-94. [CrossRef]

17. Mooney, M. The viscosity of a concentrated suspension of spherical particles. J. Colloid Interface Sci. 1951, 6, 162-170. [CrossRef]

18. Gómez-Merino, A.I.; Rubio-Hernández, F.J.; Velázquez-Navarro, J.F.; Aguiar, J.; Jiménez-Agredano, C. Study of the aggregation state of anatase water nanofluids using rheological and DLS methods. Ceramics Int. 2014, 40, 14045-14050. [CrossRef]

19. Quincke, G. Ueber die Fortführung materieller Theilchen durch strömende Elektricität. Ann. Phys. 1861, 189, 513-598. [CrossRef] 
20. Helmholtz, H. Studien über elektrische Grenzschichten. Ann. Phys. Chem. 1879, 243, 337-382. [CrossRef]

21. Gouy, G. Sur la constitution de la charge électrique à la surface d'un électrolyte. J. Phys. Theor. Appl. 1910, 9, 457-468. [CrossRef]

22. Chapman, D.L. A contribution to the theory of electrocapillarity. Phil. Mag. 1913, 25, 475-481. [CrossRef]

23. Stern, O. Zur theorie der elektrolytischen doppleschicht. Z. Elektrochem. 1924, 30, 508-516.

24. Grahame, D.C. The electrical double layer and the theory of electrocapillarity. Chem. Rev. 1947, 41, 441-501. [CrossRef]

25. Li, S.; Hou, S. A brief review of the correlation between electrical properties and wetting behaviour in porous media. Capillarity 2019, 2, 53-56. [CrossRef]

26. Hunter, R.J. Zeta Potential in Colloid Science: Principles and Applications; Academic Press Inc.: New York, NY, USA, 1981; pp. 59-124.

27. Smoluchowski, M. Elektrische Endosmose und Strömungsströme. In Handbuch der Electrizität un des Magnetismus; Graetz, L., Barth, J.A., Eds.; Verlag: Leipzig, Germany, 1914; Volume 2, pp. 366-428.

28. Hückel, E. Die kataphorese der kugel. Physik Z. 1924, 25, 204-210.

29. Henry, D.C. The Cathaphoresis of Suspended particles. Part I.-The Equation of Cataphoresis. Proc. R. Soc. Lond. A 1931, $133,106-129$.

30. Smoluchowski, M. Versuch einer mathematischen Theorie der koagulation kinetic kolloider losungen. Z. Phys. Chem. 1918, 93, 129-168.

31. Rice, C.L.; Whitehead, R. Electrokinetic flow in a narrow cylindrical capillary. J. Phys. Chem. 1965, 69, 4017-4024. [CrossRef]

32. Levine, S.; Marriott, J.R.; Neale, G.; Epstein, N. Theory of electrokinetic flow in fine cylindrical capillaries at high zeta-potentials. J. Colloid Interface Sci. 1975, 52, 136-149. [CrossRef]

33. Nieves, F.J.; Rubio-Hernández, F.J.; Hidalgo-Alvarez, R. Some experimental data concerning the effect of the composition of liquid mixtures (methanol-ethanol) on the electrophoretic coefficient. J. Non-Equilib. Thermodyn. 1988, 13, 373-384.

34. Rubio-Hernández, F.J.; Carrique, F.; Ruiz-Reina, E. The primary electroviscous effect in colloidal suspensions. Adv. Colloid Interface Sci. 2004, 107, 51-60. [CrossRef]

35. Ohshima, H. Theory of Colloid and Interfacial Electric Phenomena; Elsevier Academic Press Inc.: San Diego, CA, USA, 2006; pp. 266-282.

36. Jing, D.; Pan, Y. Electroviscous effect and convective heat transfer of pressure-driven flow through microtubes with surface charge-dependent slip. Int. J. Heat Mass Transf. 2016, 101, 648-655. [CrossRef]

37. Yeh, L.H.; Ma, Y.; Xue, S.; Qian, A. Electroviscous effect on the streaming current in a pH-regulated nanochannel. Electrochem. Commun. 2014, 48, 77-80. [CrossRef]

38. Ren, L.; Li, D.; Qu, W. Electro-Viscous Effects on Liquid Flow in Microchannels. J. Colloid Interface Sci. 2001, 233, 12-22. [CrossRef]

39. Chun, M.S.; Kwak, H.W. Electrokinetic flow and electroviscous effect in a charged slit-like microfluidic channel with nonlinear Poisson-Boltzmann field. Korea-Aust. Rheol. J. 2003, 15, 83-90.

40. Burgreen, D.; Nakache, F.R. Electrokinetic flow in ultrafine capillary slits. J. Phys. Chem. 1964, 68, 1084-1091. [CrossRef]

41. Bowen, W.R.; Jenner, F. Electroviscous effects in charged capillaries. J. Colloid Interface Sci. 1995, 173, 388-395. [CrossRef]

42. Xuan, X. Streaming potential and electroviscous effect in heterogeneous microchannels. Microfluid Nanofluid 2008, 4, 457-462. [CrossRef]

43. Jing, D.; Bhushan, B. Electroviscous effect on fluid drag in a microchannel with large zeta potential. Beilstein J. Nanotechnol. 2015, 6, 2207-2216. [CrossRef] [PubMed]

44. Mo, X.; Hu, X. Electroviscous effect on pressure driven flow and related heat transfer in microchannels with surface chemical reaction. Int. J. Heat Mass Transf. 2019, 130, 813-820. [CrossRef]

45. Prigogine, I. Introduction to the Thermodynamics of Irreversible Processes; Wiley \& Sons: New York, NY, USA, 1968.

46. Overbeek, J.Th.G. Phenomenology of lyophobic systems. Kruyt, H.R., Ed.; In Colloid Science; Elsevier: New York, NY, USA, 1952.

47. Rubio-Hernández, F.J. Electrokinetic transport coefficients of methanol-water mixtures through polystyrene plugs: Concentration dependence of phenomenological coefficients. J. Non-Equilib. Thermodyn. 1992, 17, 333-341. [CrossRef]

48. Rubio-Hernández, F.J. Electroviscous effect in porous polystyrene plugs. J. Non-Equilib. Thermodyn. 1993, 18, 195-199. [CrossRef]

49. Qiu, Y.R.; Qi, J. Electrokinetic characterization of poly (vinyl butyral) hollow fiber membranes by streaming potential and electroviscous effect. J. Membr. Sci. 2013, 425-426, 71-76. [CrossRef]

50. Sbaï, M.; Fievet, P.; Szymczyk, A.; Aoubiza, B.; Vidonne, A.; Foissy, A. Streaming potential, electroviscous effect, pore conductivity and membrane potential for the determination of the surface potential of a ceramic ultrafiltration membrane. J. Membrane Sci. 2003, 215, 1-9. [CrossRef]

51. Huisman, I.H.; Prádanos, P.; Hernández, A. Electrokinetic characterization of ultrafiltration membranes by streaming potential, electroviscous effect, and salt retention. J. Membr. Sci. 2000, 178, 55-64. [CrossRef]

52. Huisman, I.H.; Prádanos, P.; Calvo, J.I.; Hernández, A. Electroviscous effects, streaming potential, and zeta potential in polycarbonate track-etched membranes. J. Membr. Sci. 2000, 178, 79-92. [CrossRef]

53. Farsi, A.; Boffa, V.; Christensen, M.L. Electroviscous effects in ceramic nanofiltration membranes. Chem. Phys. Chem. 2015, 16, 3397-3407. [CrossRef]

54. Bharti, R.P.; Harvie, D.J.E.; Davidson, M.R. Electroviscous effects in steady fully developed flow of a power-law liquid through a cylindrical microchannel. Int. J. Heat Fluid Flow 2009, 30, 804-811. [CrossRef]

55. Tang, G.H.; Ye, P.X.; Tao, W.Q. Electroviscous effect on non-Newtonian fluid flow in microchannels. J. Non-Newton. Fluid Mech. 2010, 165, 435-440. [CrossRef] 
56. Babaie, A.; Sadeghi, A.; Saidi, M.H. Combined electroosmotically and pressure driven flow of power-law fluids in a slit microchannel. J. Non-Newton. Fluid Mech. 2011, 166, 792-798. [CrossRef]

57. Tang, G.H. Non-Newtonian flow in microporous structures under the electroviscous effect. J. Non-Newton. Fluid Mech. 2011, 166, 875-881. [CrossRef]

58. Davidson, M.R.; Barthi, R.; Harvie, D.J.E. Electroviscous effects in a Carreau liquid flowing through a cylindrical microfluidic contraction. Chem. Eng. Sci. 2010, 65, 6259-6269. [CrossRef]

59. Shamshiri, M.; Khazaeli, R.; Ashrafizaadeh, M.; Mortazavi, S. Electroviscous and thermal effects on non-Newtonian liquid flows through microchannels. J. Non-Newton. Fluid Mech. 2012, 173-174, 1-12. [CrossRef]

60. Cheng, Z.; Ning, Z.; Dai, S. The electroviscous flow of non-Newtonian fluids in microtubes and implications for nonlinear flow in porous media. J. Hydrol. 2020, 590, 125224. [CrossRef] 